\title{
Patrimoine littéraire en ligne: la renaissance du lecteur?, D. Pety (dir.)
}

\section{Roberta Sapino}

\section{(2) OpenEdition}

1 Journals

\section{Edizione digitale}

URL: http://journals.openedition.org/studifrancesi/10102

DOI: 10.4000/studifrancesi. 10102

ISSN: 2421-5856

\section{Editore}

Rosenberg \& Sellier

\section{Edizione cartacea}

Data di pubblicazione: 1 août 2017

Paginazione: 422-423

ISSN: 0039-2944

\section{Notizia bibliografica digitale}

Roberta Sapino, «Patrimoine littéraire en ligne: la renaissance du lecteur?, D. Pety (dir.)», Studi Francesi

[Online], 182 (LXI | II) | 2017, online dal 01 août 2017, consultato il 07 janvier 2021. URL: http:// journals.openedition.org/studifrancesi/10102 ; DOI: https://doi.org/10.4000/studifrancesi.10102

Questo documento è stato generato automaticamente il 7 janvier 2021.

\section{(c) $(1) \odot \odot$}

Studi Francesi è distribuita con Licenza Creative Commons Attribuzione - Non commerciale - Non opere derivate 4.0 Internazionale. 


\title{
Patrimoine littéraire en ligne: la renaissance du lecteur?, D. Pety (dir.)
}

\author{
Roberta Sapino
}

\section{NOTIZIA}

Patrimoine littéraire en ligne: la renaissance du lecteur?, sous la direction de Dominique PETY, Chambéry, Université de Savoie-Mont Blanc, 2015, 141 pp.

1 Il volume presenta gli atti della giornata di studi “Corpus littéraires numérisés: la place $\mathrm{du}$ sujet lecteur et usager" tenutasi nel novembre 2014 all'Université de Savoie-Mont Blanc, e si colloca nel contesto della riflessione sulle riconfigurazioni della lettura e del testo letterario nell'era digitale. La diffusione di supporti digitali complessi e diversificati, osserva Dominique PETY in apertura al volume (Introduction, pp. 7-21), favorisce lo sviluppo di un nuovo umanesimo fondato sulla dimensione partecipativa della cultura. Una delle conseguenze principali, continua Pety, è il rimodellamento delle gerarchie che regolano i sistemi di produzione e di diffusione del sapere, sia a livello istituzionale sia a livello storico-letterario: la conformazione interattiva e intertestuale dei supporti recenti favorisce infatti non solo la partecipazione di contributori esterni alle istituzioni tradizionali, ma anche la valorizzazione di autori e testi considerati come minori e spesso relegati in nota $o$ in appendice delle pubblicazioni cartacee. Sulla scia delle teorie di Eco sull'opera aperta, il volume propone quindi una serie di riflessioni, tutte saldamente fondate su esperienze concrete, sulle riconfigurazioni attuali della figura del lettore e sul suo progressivo avvicinamento al ruolo autoriale.

2 Le due parti che compongono il libro sono organizzate cronologicamente secondo la datazione dei testi oggetto di digitalizzazione. La prima («L'héritage latin et l'âge classique») si apre con un contributo collettivo firmato da Michele AMBrosino, Bruno BUREAU, Gaia CASTALDI, Sara FASCIONE, Federica NICOLARDI e Christian NICOLAS, ovvero i due membri fondatori del laboratorio Hyperdonat (Université Lyon 3) e quattro studenti 
dell'università Federico II che presso il laboratorio hanno svolto un periodo di stage e formazione. Intitolato Hyperdonat de Lyon à Naples (pp. 25-45), l'articolo presenta un bilancio approfondito delle attività svolte, dimostrando non solo le potenzialità didattiche del progetto, ma anche la sostanziale affinità delle procedure necessarie per l'edizione cartacea e digitale di un testo. Non sulla creazione, ma sull'utilizzo critico di materiale digitale si concentra invece il contributo di Marc VuILLERMOz (L'invention du lecteur: un enjeu stratégique des préfaces de pièces de théâtre aux XVI et XVII siècles (France, Italie, Espagne), pp. 47-55). Basandosi sulle risorse contenute nel sito IdT - Les idées $d u$ théâtre, l'autore indaga lo statuto attribuito al lettore delle pièces teatrali tra il Rinascimento e l'epoca del Classicismo, e propone di ricondurre a tale momento storico-letterario l'origine della figura del lettore moderno, giudice del testo e produttore di senso. A seguire, l'intervento di Claude BOURQUI (Du corpus à l'outil herméneutique: la base de données intertextuelle "Molière 21", pp. 57-68) presenta i contenuti e le caratteristiche tecniche del sito Molière 21 e mette in luce una delle conseguenze della mutevolezza del mondo digitale: progettato nel 2006 come complemento all'edizione nella «Bibliothèque de la Pléiade» delle opere di Molière, il sito è raramente utilizzato nei modi previsti dagli ideatori, ma si svincola sempre di più dal suo corrispettivo cartaceo. Lo studio delle pratiche di lettura degli utenti del web è l'elemento alla base dell'intera concezione dell'edizione online delle Nouvelles Nouvelles di Donneau de Visé, presentata nell'articolo di Christophe schuwey (Faire lire les "Nouvelles Nouvelles" de Donneau de Visé: défis et opportunités d'une édition en ligne, pp. 69-84). Avendo notato la somiglianza tra le pratiche di lettura sul web e i modi di fruizione della letteratura mondana nel Seicento, i curatori delle Nouvelles Nouvelles hanno elaborato una struttura di schede e rimandi in cui il discorso critico interviene soltanto laddove il lettore lo richiede, e sempre con la massima sintesi ed efficacia. In questo modo, il sito consente non solo di avvicinare il pubblico a un testo poco conosciuto, ma anche di restituire al lettore un'esperienza di rapporto al testo quanto più simile a quella originale.

3 La seconda sezione è dedicata a progetti riguardanti «Archives et manuscrits du XIX siècle». Jean-Sébastien MACKE illustra le trasformazioni che ha subito ArchiZ sulla spinta dell'ampliamento e della diversificazione dell'utenza: la creazione di un gruppo di ricerca in collaborazione con il Ministero dell'Educazione Nazionale e il coinvolgimento di gruppi di studenti per la rielaborazione grafica del sito hanno reso ArchiZ sia una risorsa scientifica di eccellenza, sia uno strumento didattico e pedagogico innovativo (Les archives zoliennes: création et évolution du site Archiz en fonction de ses lecteurs et usagers, pp. 87-95). Cécile MEYNARD e Thomas LEBARBÉ testimoniano del loro lavoro all'interno di un progetto che porterà all'edizione digitale delle circa quarantamila pagine che compongono il fondo Stendhal della Bibliothèque Municipale de Grenoble. In particolare, gli autori osservano come solo l'edizione digitale permetta di assolvere al doppio compito di fornire una rappresentazione fedele dei manoscritti e di aumentarne la leggibilità. Inoltre, il sito è organizzato in modo da sollecitare l'interazione del lettore, che può crearsi un proprio corpus di pagine, partecipare alla trascrizione dei manoscritti e contribuire all'arricchimento dei contenuti (Donner à voir, à lire, à comprendre: destinataires et finalités d'une édition polymorphe des manuscrits de Stendhal, pp. 97-115). L'intervento di Stéphanie DORD-CROUSLÉ, infine, rende conto di un caso emblematico in cui il lettore è sollecitato a sostituirsi all'autore: grazie alla mobilità e all'interattività tipiche del supporto digitale, i frammenti che compongono il 
materiale preparatorio del secondo libro di Bouvard et Pécuchet possono essere classificati e ordinati liberamente, e il lettore può anche ipotizzare un finale per il romanzo flaubertiano incompiuto (Les "seconds volumes" possibles de "Bouvard et Pécuchet": l'avènement d'un lecteur auteur?, pp. 117-131).

4 In virtù della varietà e della concretezza delle esperienze presentate, il volume fornisce spunti di grande interesse soprattutto da un punto di vista metodologico. 